\title{
Categorical mistakes and moral biases in the withholding-versus-withdrawal debate
}

Author: Bjørn Hofmann

Affiliations:

Institute for the Health Sciences at the Norwegian University of Science and Technology, Gjøvik, Norway

The Centre of Medical Ethics at the University of Oslo

bjoern.hofmann@ntnu.no

Abstract:

Discrepancies between bioethical theories and guidelines on the one hand and clinical practice on the other, call for reflection. Ursin contributes to this reflection in focusing on contextual issues undermining the ceteris paribus clause of the Equivalence Thesis (ET). Wilkinson and colleagues dismiss any differences between withholding and withdrawal as irrational biases. Unfortunately the latter's approach itself appears biased and their cases support Ursin's point more than ET. The argument for ET beyond the ceteris paribus clause appears to be as biased as the argument for the non-equivalence thesis with the ceteris paribus clause applying. More work is needed to clarify the relationship between withholding and withdrawing treatment. 


\section{Introduction}

Lars Ursin gives three reasons why we may not accept the widely held Equivalence Thesis (ET) in bioethics without careful reflection and contextual qualification. First, autonomy can be compromised differently in withdrawal and withholding treatment. Second, professional responsibility may be different as well. Additionally, Ursin also points out that "to continue ongoing treatment has another status for the patient than to embark on a new treatment." (Ursin, In press)

Dominic Wilkinson, Ella Butcherine, and Julian Savulescu dismiss all three arguments based on the claim that the experienced difference between withholding and withdrawal is based on a cognitive bias they call "Withdrawal Aversion - a non-rational preference for withholding treatment over withdrawal of treatment." (D. Wilkinson, Butcherine, \& Savulescu, In press) To bolster their argument they provide two cases.

However, instead of providing rational arguments strengthening the ET, they appear to weaken ET by biases of their own. Moreover, they tend to support Ursin's arguments with their cases. Both contributions clearly show that more work is needed. In particular in the field of investigating clinicians' conceptions of difference in the cases of withdrawal from those of withholding treatment.

\section{The unhelpful cases of Paula and Theo}

Ursin points out that "the ceteris paribus clause of the ET is hard to meet," and thus, that the ET may have much less relevance in practical clinical cases than its prominence in guidelines suggest. Instead of addressing this issue, Wilkinson and colleagues provide two cases that appear to support Ursin's point more than their own.

In the case of Paula (an extremely preterm infant with $40 \%$ chance of survival and subsequent 15 $20 \%$ chance of severe neurological impairment) withdrawal is not acceptable (as is to withhold) because of the $\mathrm{ET}$, but because the service is preference-sensitive and the parents have a clear preference for terminating the treatment (it has been commenced and after gaining information and having time to reflect). There is no ceteris paribus condition.

In the case of Theo (a 6-month-old infant with a congenital genetic disorder where there exists a new life-prolonging but highly expensive medicine that is in very short supply and that has specific inclusion criteria) the withdrawal is not acceptable due to the ET either. It is because the inclusion criteria are not met anymore. Hence, there is no ceteris paribus condition in this case either.

Wilkinson and colleagues argue that "the Equivalence Thesis is best understood as a statement about comparative permissibility: if it is permissible to WH treatment it would also be permissible to WD the same treatment (if already started, and all other things being equal)." Therefore, they argue, it would be permissible to stop treatment both in the cases of Paula and Theo.

First, this is not a demonstration of the ET, as things are not equal. For the same reasons it does not undermine the non-equivalence thesis either. People defending the non-equivalence thesis could agree to withdraw treatments in the case of Paula and Theo due to morally relevant differences in the cases without being irrational. Wilkinson and colleagues would have to show that health professionals would differ with respect to withholding and withdrawal in morally equivalent cases in order to show that the non-equivalence thesis is wrong. 
Second, the cases rather underscore Ursin's point: context may provide differences. While ET is a theoretically sound stance, it may rarely apply in practice, and thus be less relevant than assumed in guidelines. Instead of taking Ursin's main point into account and investigate whether there are reasonable differences between cases of withholding and withdrawal, Wilkinson and colleagues label opposing views as "irrational" and ruled by flawed intuitions and "cognitive biases."

\section{Biases in the WH-WD-debate}

The defense of ET is itself full of biases. First, Wilkinson and colleagues use biased language. They compare "the non-equivalence intuition" to "the Equivalence Thesis" (my emphasis). Furthermore, they describe the thesis that there can be morally relevant differences between withholding and withdrawal in terms of "aversion," "bias," "prejudice," having "irrational effects on decision making" (D. Wilkinson et al., In press) and elsewhere as "omission bias" and "status quo bias"(Dominic Wilkinson \& Savulescu, 2014). While it is both legitimate and laudable to reveal irrational aspects in ethics, superficial labelling of opposing views as biased does not do the trick. For instance, the status quo bias is based on the endowment effect (Kahneman, Knetsch, \& Thaler, 1991), i.e., the effect "which causes an item to be viewed as more desirable immediately upon its becoming part of one's endowment"(Bostrom \& Ord, 2006). Explaining differences in decisions on withholding versus withdrawing on psychological effects demonstrated with experiments with mugs, chocolate bars, and electric power plans or a single small vignette study with low response rate (Aberegg, Haponik, \& Terry, 2005; Dominic Wilkinson \& Savulescu, 2014) may do the trick if you are already inclined (biased) towards a specific view, but it does no heavy lifting ethically, if you are not.

Moreover, as Ursin points out, the defense of ET is motivated by a desire to make withdrawal on equal standing as withholding "even if the patient or relatives disagree."(Ursin, In press) The belief is "that it is better to withdraw than to withhold treatment in the ICU, because starting treatment can make better informed medical decision-making possible."(Ursin, In press) However, gained knowledge violates the ceteris paribus clause. The argument for ET beyond the ceteris paribus clause appears to be as biased as the argument for the non-equivalence thesis with the ceteris paribus clause applying.

Moreover, if you think it is better to start treatment and then withdraw on a later stage, then you may be subject to bias: anticipated decision regret. There seems to be no bias free zone, although it is easier to see the bias in the opponents' eyes than in your own.

\section{Categorical mistakes}

Wilkinson and colleagues argue that "intuitions have no epistemic force." However, it is far from clear whether intuitions are categorically comparable to evidence (Allman \& Woodward, 2008; Pust, 2012). Moreover, it can be argued that moral theories are unjustified altogether because they cannot be tested and confirmed (Harman, 1977). Moral intuitions, as presented in cases such as for example Thompson's transplant case, are used to prima facie undermine ethical theories, and thus are important for ethics.

In the case of withholding and withdrawing medical treatment Wilkinson and colleagues have a strong intuition that we should revise our intuitions and not our theory (D. Wilkinson et al., In press). 
This intuition bolsters their own conception that "the arguments in favour of Equivalence are strong, clear and hard to reject" (Dominic Wilkinson \& Savulescu, 2014) but blocks their ability to taking Ursin's arguments seriously.

The point here is not to enter the debate on moral intuition, but more modestly to highlight that much more work needs to be done to dismiss intuitions in ethics than Wilkinson and colleagues provide.

\section{Looking for differences}

Instead of bluntly labelling experienced differences between withholding and withdrawal of treatment as irrational biases, we should look for potential explanations, as Ursin does (Ursin, In press). When there are no differences, we should certainly adhere to the ET. Here Wilkinson and colleagues' Equivalence Test is very helpful (D. Wilkinson et al., In press). However, where there are differences, we should investigate whether they are morally relevant.

For example, due to differences in time and information, there may be an epistemic asymmetry: we know more after having started treatment. Hence, there may be a morally relevant difference in epistemic uncertainty.

Another potential explanation for the experienced difference is the commitment of health professionals when having started a treatment. Withdrawing treatment may seem arbitrary to patients and proxies, even when justified from a purely medical, economic, or ethical (ET) point of view. Finding ways to address this commitment and avoid experiences of arbitrariness may be more effective approaches than hammering on with a theoretical thesis.

Another relevant issue may be found in basic asymmetries in ethics (Tranöy, 1967), e.g., that actions of prevention of death may be different than action of cessation of life. There is more ground to investigate before one can dismiss all experienced differences as irrational biases.

\section{Conclusion}

Additional studies of the relationship between withholding and withdrawing treatment are most welcome, and the present studies (Ursin, In press; D. Wilkinson et al., In press) are valuable contributions. Ursin points to an important mismatch between the theory (the Equivalence Thesis) well expressed in guidelines and the situations experienced by professionals in clinical practice. Wilkinson and colleagues provide a useful Equivalence Test.

However, if the conditions where the Equivalence Thesis apply are much less frequent due to morally relevant differences breaching with the ceteris paribus clause than thought, the Thesis may be less important than its place in theory and guidelines suggest. If this is correct, we should study the differences more closely than previously done. In particular, to rhetorically label such differences irrational and call moral intuitions "cognitive biases" may be a categorical mistake or in itself be a "moral bias." The argument for ET beyond the ceteris paribus clause appears to be as biased as the argument for the non-equivalence thesis with the ceteris paribus clause applying.

If a well-founded theory for applied ethics is not applicable to clinical cases due to contextual insensitivity, one may consider refining the theory in order to address the contextual issues instead 
of dismissing them as biases. Ursin as well as Wilkinson and colleagues clearly show that more work is needed.

\section{References}

Aberegg, S. K., Haponik, E. F., \& Terry, P. B. (2005). Omission bias and decision making in pulmonary and critical care medicine. Chest, 128(3), 1497-1505.

Allman, J., \& Woodward, J. (2008). What are moral intuitions and why should we care about them? A neurobiological perspective. Philosophical Issues, 18(1), 164-185.

Bostrom, N., \& Ord, T. (2006). The reversal test: eliminating status quo bias in applied ethics. Ethics, $116(4), 656-679$.

Harman, G. (1977). The Nature of Morality an Intorduction to Ethics. Oxford: Oxford University Press.

Kahneman, D., Knetsch, J. L., \& Thaler, R. H. (1991). Anomalies: The Endowment Effect, Loss Aversion, and Status Quo Bias. Journal of Economic Perspectives, 5(1), 193-206. doi:doi: 10.1257/jep.5.1.193

Pust, J. (2012). Intuition.

Tranöy, K. E. (1967). Asymmetries in ethics: on the structure of a general theory of ethics. Inquiry, 10(1-4), 351-372.

Ursin, L. (In press). Withholding and Withdrawing Life-Sustaining Treatment: Ethically Equivalent? AJOB.

Wilkinson, D., Butcherine, E., \& Savulescu, J. (In press). Withdrawal Aversion and the Equivalence Test. AJOB.

Wilkinson, D., \& Savulescu, J. (2014). A costly separation between withdrawing and withholding treatment in intensive care. Bioethics, 28(3), 127-137. 